\title{
A PRODUÇÃO DE SAL MARINHO NO BRASIL E SUA CORRELAÇÃO COM A PLUVIOMETRIA ANUAL E O CRESCIMENTO ECONÔMICO NACIONAL
}

\author{
Marco Túlio Mendonça Diniz \\ Universidade Federal do Rio Grande do Norte - UFRN \\ Docente do Programa de Pós-Graduação em Geografia - PROPGEO \\ tuliogeografia@gmail.com \\ Fábio Perdigão Vasconcelos \\ Universidade Estadual do Ceará - UECE \\ Docente do Programa de Pós -Graduação em Geografia - PROPGEO \\ fabioperdigao@gmail.com
}

\begin{abstract}
RESUMO
Existem dados da produção de sal marinho a partir do ano de 1938, neste trabalho estes dados foram analisados ano a ano, com destaque para períodos de crescimento, decréscimo e estagnação. Estes períodos foram correlacionados com transformações econômicas e nos espaços de produção da atividade s alineira. As transformações es paciais foram analisadas dos pontos de vista social e natural. $O$ método estatístico utilizado para correlacionar as variáveis pluviometria, PIB do Brasil e produção nacional de sal marinho foi o Coeficiente de Correlação de Pearson. A atividade salineira tem seu crescimento relacionado com o cres cime nto econômico do país e com a modernização pela qual o setor passou entre os anos 1960 e 1970 quando o Rio Grande do Norte se consolidou como maior produtor nacional. Exis te forte influencia do meio natural com o desenvolvimento da atividade salineira, que é concentrada, principalmente, em determinada porção do Nordes te Setentrional, nessa região a atividade é influenciada pela variação interanual do regime de chuvas.
\end{abstract}

Palavras-chave: Relação sociedade-natureza. "Influencismo" geográfico. Geografia Econômica.

\section{THE SEA SALT PRODUCTION IN BRAZIL AND THE INFLUENCE OF RELATIONSHIP WITH PLUVIOMETRY AND THE NATIONAL ECONOMIC GROWTH}

\begin{abstract}
There are data of sea salt production from the year 1938, this work these data were analyzed from year to year, making emphasis on periods of growth, decline and stagnation. These periods were related to economic changes and spaces of production of the salt activity. The spatial transformations were analyzed from s o cial and natural points of view. The statistical method used to relate the variables rainfall, Brazil's GDP and domestic production of sea salt was the Pearson correlation coefficient. The Salt work activity has his growth related with economic growth and modernization in which the industry experienced between the 1960s and 1970s when Rio Grande do Norte has es tablis hed its elf as the largest national producer. There is a strong influence of the natural environment with the development of the salt activity, which is concentrated mainly in a certain portion of the northern Northeast of Brazil, in this region the activity is influenced by the interannual variation of rainfall.
\end{abstract}

Keywords: Relationship between society and nature. Geographical "influencism". Economic geography.

\section{INTRODUÇÃO}

A utilização do sal marinho no Brasil precede à colonização europeia, ao longo dos anos ele foi produzido principalmente em estados do Nordeste Setentrional (mais de $90 \%$ no Ceará e no Rio Grande do Norte) e no Rio de Janeiro, ao redor da Lagoa de Araruama (Figura 1).

\begin{tabular}{llllll}
\hline Caminhos de Geografia & Uberlândia -MG & v. 20, n.69 & Março/2019 & p. 20-35 & Página 20
\end{tabular}




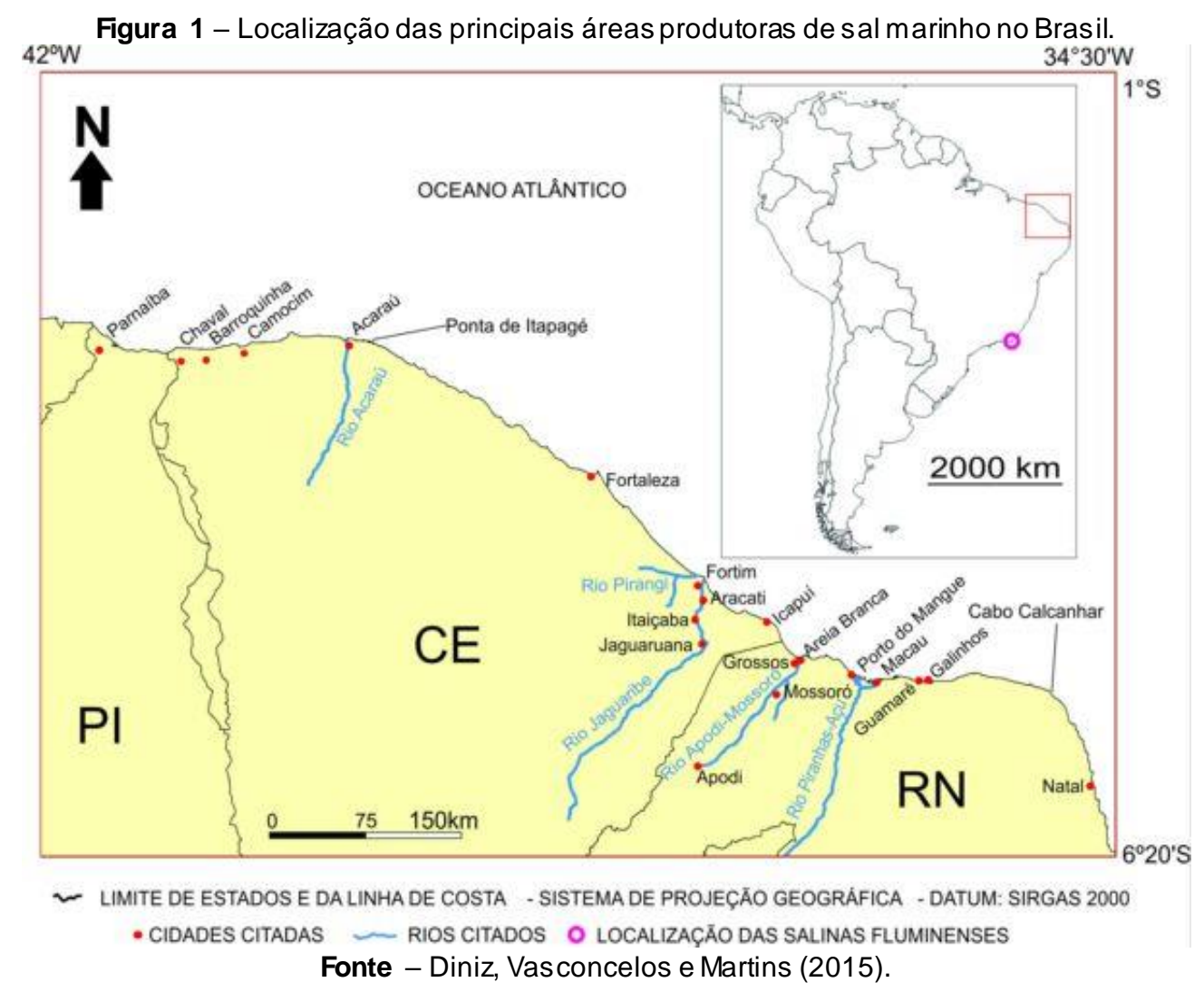

Apenas após à vinda da Família Real Portuguesa ao Brasil é que esta atividade foi estimulada de forma oficial, esta história pode ser melhor identificada na contextualização dos fatos históricos relevantes para a construção da economia salineira do Brasil apontados por Diniz e Vasconcelos (2016) e no artigo que trata da inovação tecnológica pela qual passou esta economia (DINIZ; VASCONCELOS; MARTINS, 2015). Este artigo teve como objetivo prosseguir na análise espacial do histórico da produção do sal marinho no Brasil iniciada por Diniz e Vasconcelos (2016), só que nesta oportunidade o enfoque foi o de analisar os dados estatísticos disponíveis sobre a produção de sal marinho, correlacionando a produção com o crescimento econômico do país e com a taxa de precipitação pluviométrica anual registrada nas principais áreas produtoras.

\section{METODOLOGIA}

Apenas a partir do ano de 1938 no Anuário Estatístico do Brasil, editado pelo Instituto Brasileiro de Geografia e Estatística - IBGE, passou a constar dados da produção brasileira de sal. Esses dados estão disponíveis de forma detalhada por unidade da federação, desde 1938 até 1990, quando passaram a ser fornecidos pelo Sumário Mineral Brasileiro e pelo Anuário Mineral Brasileiro, ambos editados pelo Departamento Nacional de Produção Mineral - DNPM.

Com base nestes dados foi feita uma análise descritiva das estatísticas da produção de sal marinho no Brasil, correlacionando essa produção com eventos econômicos, políticos e a precipitação pluviométrica anual ocorridos no Brasil e na região, dentre os quais, a criação do Instituto Nacional do Sal e a estiagem no Nordeste.

A correlação entre produção de sal marinho e os totais pluviométricos anuais, bem como a correlação entre a produção de sal marinho e o crescimento do Produto Interno Bruto (PIB) do Brasil foram feitas de modo estatístico, utilizando o Coeficiente de Correlação Linear de Pearson ( $r$. 


\section{Do coeficiente de correlação linear de pearson ( $r$ )}

Para a verificação da relação entre duas variáveis é comum em pesquisas a utilização do Coeficiente de Correlação Linear de Pearson (r). Esse coeficiente é utilizado em estatística para designar a força que mantém unidos dois conjuntos de valores, ou seja, para estabelecer - grau de correlação positiva ou negativa entre duas variáveis. Uma vez caracterizada essa relação, procuramos descrevê-la de forma matemática, utilizando uma função. Para visualização cartesiana da relação, as variáveis são colocadas num diagrama de dispersão.

Duas variáveis apresentam correlação linear quando os pontos num diagrama de dispersão se aproximam de uma reta.

Essa correlação pode ser positiva (para valores crescentes de $X$, há uma tendência a valores também crescentes de $Y$ ) ou negativa (para valores crescentes de $X$, a tendência é observarem-se valores decrescentes de $Y$ ) (NAGHETTINI; PINTO, 2007, p. 357).

As correlações lineares positivas e negativas encontram-se ilustradas na Figura 2:

Figura 2 - Diagramas de Correlações Lineares Positivas e Negativas.
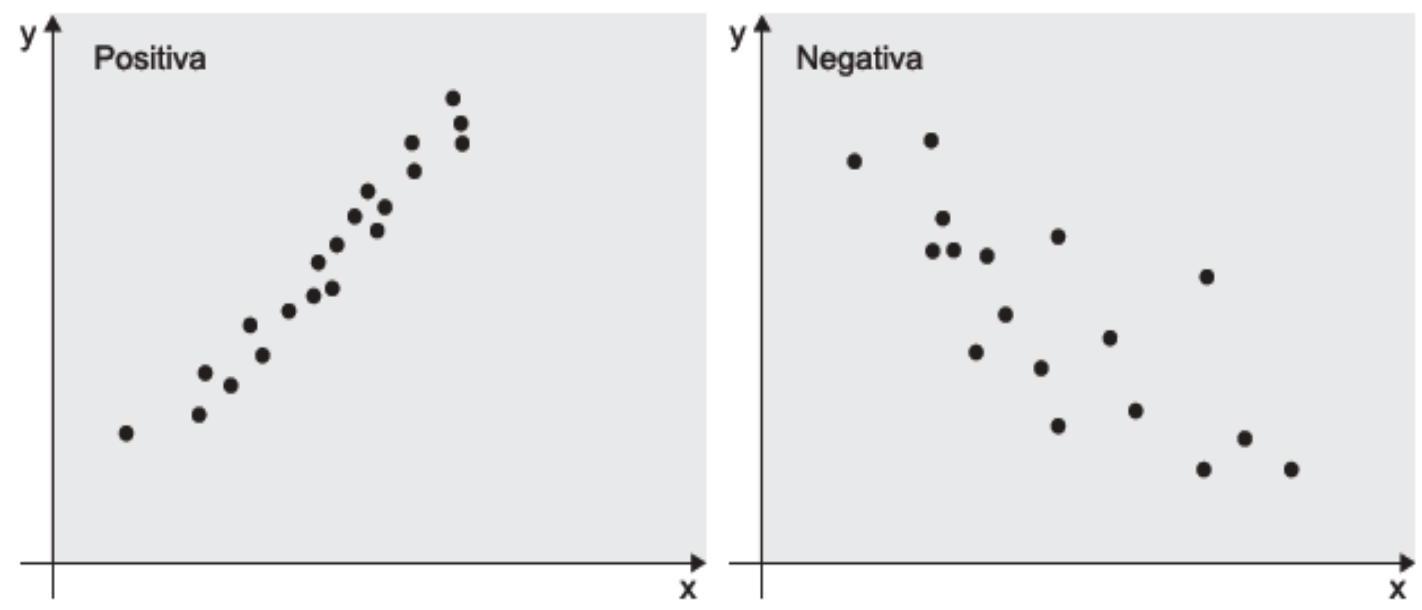

Fonte - NAGHETTINI; PINTO, 2007.

De acordo com Souza (online), o coeficiente de correlação linear é representado por:

$$
r=\frac{L_{x y}}{s_{x^{5} y}}
$$

Onde:

$c_{x y}$ - Covariância ou variância conjunta das variáveis $\mathrm{X}$ e $\mathrm{Y}$;

$S_{x}$ - Desvio padrão da variável $X$;

$S_{y}$ - Desvio padrão da variável $Y$.

De acordo com Souza (online), a relação entre o coeficiente de Pearson e a correlação é definida pelos seguintes parâmetros (Quadro 1): 
Quadro 1 - Grau de correlação entre as variáveis

\begin{tabular}{|c|c|}
\hline Coeficiente de correlação & Correlação \\
\hline$r=1$ & Perfeita positiva \\
\hline $0,8 \leq r<1$ & Forte positiva \\
\hline $0,5 \leq r<0,8$ & Fraca positiva \\
\hline $0,1 \leq r<0,5$ & Ínfima positiva \\
\hline $0<r<0,1$ & Nula \\
\hline 0 & Ínfima Negativa \\
\hline$-0,1<r<0$ & Fraca Negativa \\
\hline$-0,1<r \leq-0,5$ & Moderada Negativa \\
\hline$-0,8<r \leq-0,5$ & Forte Negativa \\
\hline$-1<r \leq-0,8$ & Perfeita Negativa \\
\hline$r=-1$ &
\end{tabular}

Fonte - Souza online.

Foram realizadas correlações de duas formas:

A primeira correlação foi feita cruzando-se dados da produção de sal marinho do Brasil e por unidade da federação (dados foram fornecidos pelo IBGE e pelo DNPM) com a taxa de precipitação em alguns postos pluviométricos do Nordeste (dados da SUDENE, 1990). A escolha dos postos se deu levando em consideração, primeiramente, a proximidade dessas com as áreas produtores, e ainda, de acordo com a disponibilidade de dados ano a ano, já que nem todos têm dados para todos os anos analisados. Considerando a disponibilidade de dados, o período considerado nesta análise foi os anos de 1938 a 2013.

A segunda correlação foi feita cruzando-se os mesmos dados da produção de sal marinho do Brasil e por unidade da federação (dados foram fornecidos pelo IBGE e pelo DNPM) com a taxa de crescimento do PIB do Brasil (fornecida pelo IBGE). Considerando a disponibilidade de dados, o período considerado nesta análise também foi os anos de 1938 a 2013. Os cálculos e os gráficos de ambas as análises foram realizados no software Microsoft Excel 2010.

Os dados da produção salineira analisados consideram os três maiores produtores, que respondem atualmente por $99 \%$ do sal marinho produzido no país, os dados foram agrupados em dois períodos temporais, o primeiro deles de 1938 a 1970 (Gráfico 1), este foi delimitado, de forma aproximada, entre o início do fornecimento de dados oficiais confiáveis e detalhados por unidade da federação (1938), e o fim das salinas artesanais e início do empreendimento da mecanização da produção de sal marinho no Nordeste, que ocorreu no final dos anos 1960 (DINIZ; VASCONCELOS, 2016). Outro fato importante foi a construção do Porto llha de Areia Branca/RN, inaugurado no ano de 1974.

O segundo período temporal de agrupamento dos dados da produção salineira vai do ano de 1971 até 2013 (Gráfico 2), na década de 1970 houveram as transformações na economia salineira potiguar.

Considerando que nos estados do Ceará e Rio Grande do Norte a atividade das salinas solares sofre maior influência das variações interanuais características do Clima (Tropical de Zona Equatorial de subtipo semiárido) da região foram feitas correlações entre a produção anual e total pluviométrico anual registrado nos postos pluviométricos da SUDENE em Jaguaruana/CE e Areia Branca/RN.

Os dados do PIB do Brasil foram retirados das Contas Nacionais, disponíveis online (IBGE, 2006; online). 
Gráfico 1 - Produção brasileira de sal marinho em toneladas nos três maiores estados produtores: Rio de Janeiro (RJ); Ceará (CE); e Rio Grande do Norte (RN) entre os anos de 1938 e 1970.

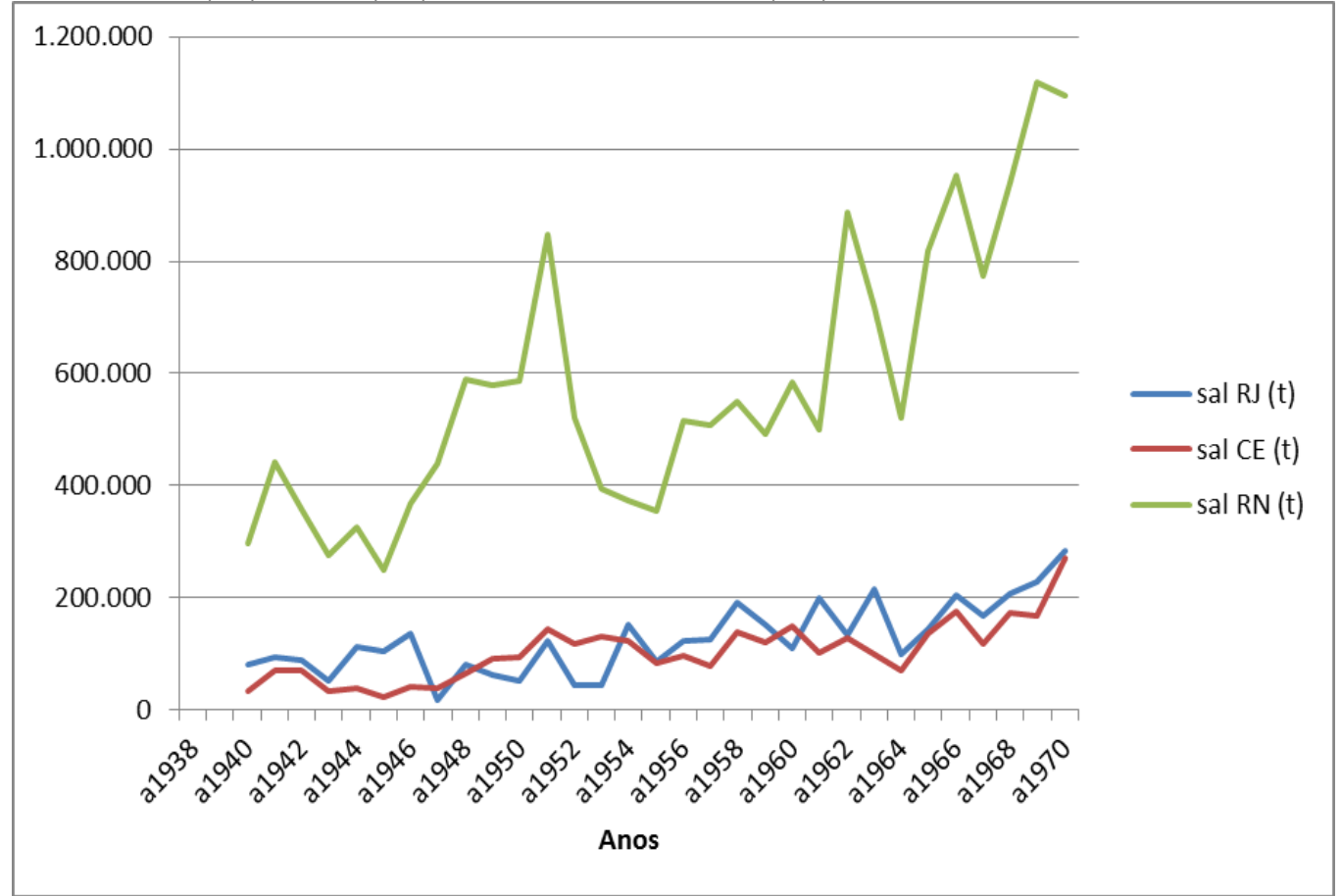

Fonte - Anuário Estatístico do Brasil - Anos de1941/1945, 1946, 1947, 1949, 1951, 1953, 1954, 1955, 1957, 1960, 1961, 1963, 1966, 1969 e 1972.

Gráfico 2 - Produção brasileira de sal marinho em toneladas nos três maiores estados produtores: Rio de Janeiro (RJ); Ceará (CE); e Rio Grande do Norte (RN) entre os anos de 1971 e 2013.

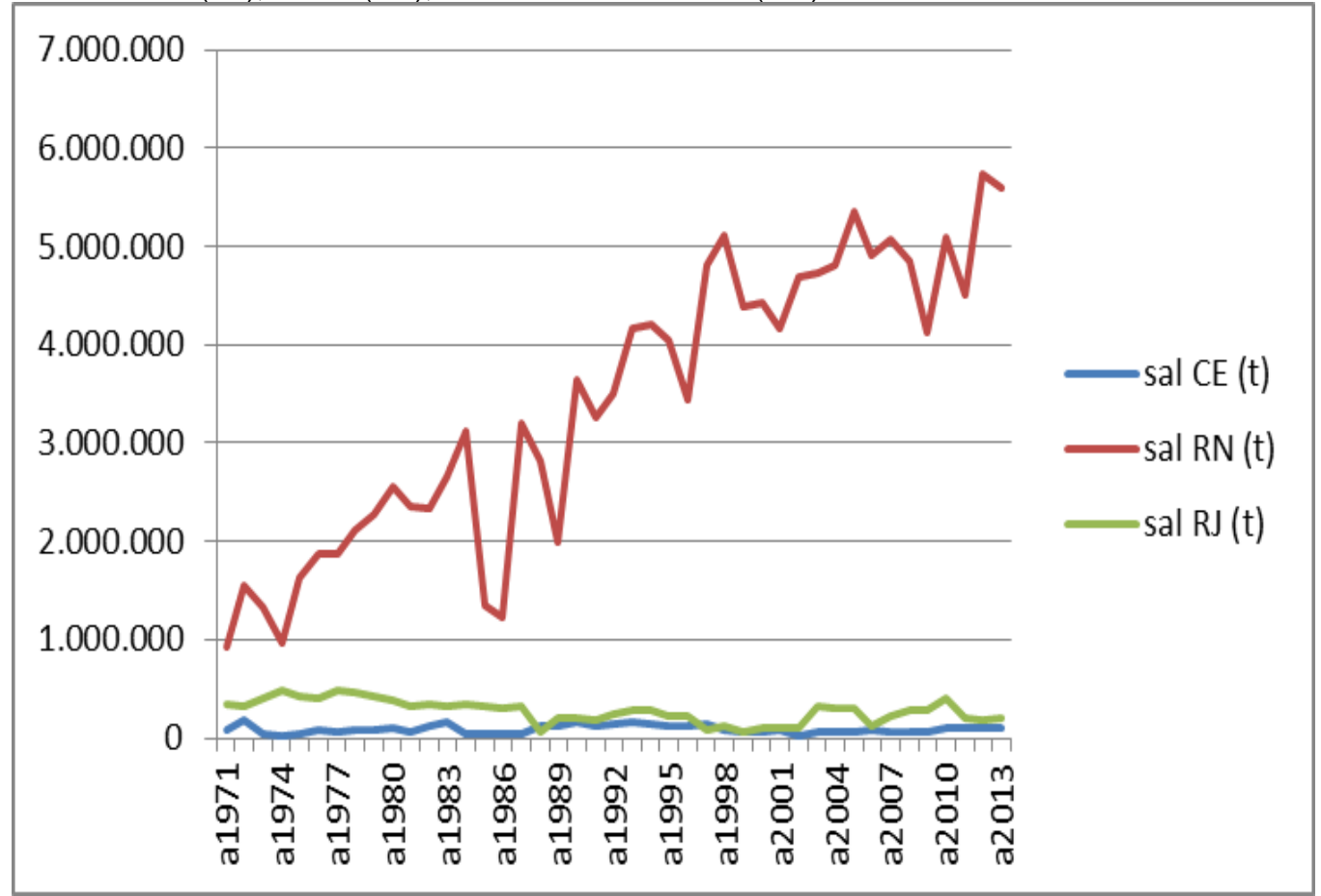

Fonte - Anuário Estatístico do Brasil - Anos de 1972, 1975, 1978, 1981, 1987/1988, 1991, 1994 e 1997; Sumário Mineral Brasileiro - anos de 1992 a 2011; e Anuário Mineral Brasileiro de 1995 e 2004. 


\section{RESULTADOS E DISCUSSÃO}

Numa rápida análise do gráfico 1 é possível observar que durante o período entre 1938 e 1970 a produção de sal marinho dos Estados do Rio Grande do Norte, Ceará e Rio de Janeiro têm tendência geral de crescimento, com oscilações naturais de ano a ano, contudo, após os anos 1970, esse crescimento se deu de uma forma bem diferente para os três maiores estados produtores.

Para a análise dos dados da produção de sal marinho, desde a década de 1970, até os dias de hoje (Gráfico 2), tem-se que considerar pelo menos três fatores sociais de suma importância: as ações empreendedoras do processo de mecanização das salinas potiguares (DINIZ, VASCONCELOS; MARTINS, 2015); a construção do Porto-llha de Areia Branca; e ainda as oscilações da economia brasileira com o auge do "Milagre Econômico Brasileiro" e seu posterior declínio com vários anos de estagnação do crescimento econômico. Em uma primeira análise, é possível perceber que entre os anos de 1970 e 2014 apenas o Estado do Rio Grande do Norte viu sua produção crescer e de forma exponencial, enquanto que no Ceará e no Rio de Janeiro houve declínio da indústria de sal marinho.

\section{Correlação estatística entre precipitação pluviométrica anual e produção de sal marinho}

A produção de sal marinho sofre influência direta da precipitação pluviométrica. Os anos mais secos tendem a ser de maior produção de sal marinho, assim como nos anos mais chuvosos a produção de sal marinho tende a ser menor. Para expressar matematicamente esta relação fizeram-se cálculos do Coeficiente de Correlação Linear de Pearson (r).

Os dados foram agrupados em período de alguns anos para que seja diminuído o efe ito do crescimento vegetativo da produção ao longo dos quase 80 anos para os quais há informações de precipitação e produção de sal marinho por estado. Foram feitos os cálculos para os dois maiores produtores nordestinos, dado que esses estão mais expostos às oscilações climáticas do clima semiárido da região. Os dados de pluviometria utilizados nos cálculos são do posto pluviométrico da SUDENE de Jaguaruana para a correlação com a produção cearense, e do posto da SUDENE, em Areia Branca, para a correlação com a produção do Rio Grande do Norte.

No Rio Janeiro as variações interanuais de precipitação foram irrisórias e não tiveram influência sobre a produção no período analisado.

Os dados do Quadro 2 nos mostram que existiu de moderada à forte correlação linear negativa entre a produção de sal marinho do Ceará e as taxas de precipitação anual do posto pluviométrico de Jaguaruana nos períodos de 1938-1948, 1949-1958, 1959-1968, 1969-1978 e 1979-1988. As salinas do Ceará sempre produziram menos em comparação às do estado vizinho, sua produção nunca representou mais do que $18 \%$ do total nacional, devido a seu menor potencial produtivo (natural e de infraestrutura instalada). Em relação ao Rio Grande do Norte, os produtores de sal marinho do Ceará sempre estiveram mais dependentes das oscilações do clima. Na maioria dos anos, conseguiam vender toda a sua (pequena) produção, não fazendo muitos estoques, daí a maior correlação entre clima e produção.

A partir da década de 1990, aprofunda-se a crise da economia salineira do Ceará. Após 1989, existe uma fraca correlação negativa entre produção e pluviometria, já que o gráfico da produção de sal marinho do Ceará tem tido uma linha geral de decréscimo.

\begin{tabular}{llllll}
\hline Caminhos de Geografia & Uberlândia - MG & v. 20, n. 69 & Março/2019 & p. 20-35 & Página 25
\end{tabular}


Quadro 2 - Coeficiente de correlação linear de Pearson (r) entre a produção de sal marinho no RN e a taxa de precipitação pluviométrica anual em Jaguaruana entre os anos de 1938 e 2008; e entre a produção de sal marinho no RN e a taxa de precipitação pluviométrica anual em Areia Branca entre os anos de 1938 e 2008.

\begin{tabular}{|c|c|c|}
\hline Períodos & r no RN & r no CE \\
\hline $\mathbf{1 9 3 8 - 1 9 4 8}$ & $-0,13$ & $-0,84$ \\
\hline $\mathbf{1 9 4 9 - 1 9 5 8}$ & $-0,04$ & $-0,75$ \\
\hline $\mathbf{1 9 5 9 - 1 9 6 8}$ & $-0,06$ & $-0,51$ \\
\hline $\mathbf{1 9 6 9 - 1 9 7 8}$ & $-0,46$ & $-0,76$ \\
\hline $\mathbf{1 9 7 9 - 1 9 8 8}$ & $-0,63$ & $-0,51$ \\
\hline $\mathbf{1 9 8 9 - 1 9 9 8}$ & $-0,53$ & $-0,02$ \\
\hline $\mathbf{1 9 9 9 - 2 0 0 8}$ & $-0,51$ & $-0,35$ \\
\hline
\end{tabular}

Fonte - Dados da produção de sal: Anuário Estatístico do Brasil - anos 1941/1945 a 1990; e Sumário Mineral Brasileiro de 1991 a 2009. Dados de precipitação de 1938 a 1985 em Jaguaruana e de 1938 a 1991 em Areia Branca SUDENE (1990); em Jaguaruana, de 1986 a 2008 FUNCEME (online); em Areia Branca, de 1993 a 2008 EMPARN (online). Não há dados de produção de sal para o ano de 1939. Não há dados de precipitação para ano de 1976 em Jaguaruana e 1944, 1992, 1999 e 2001 em Areia Branca.

Ao contrário do que ocorreu com o Ceará, as oscilações da produção no Rio Grande do Norte são muito mais dependentes do que acontece na economia (e na política) do país. As cotas de produção estabelecidas pelo Governo Federal até o ano de 1967 (ANDRADE, 1995) são a principal explicação para uma correlação ínfima negativa entre a produção de sal marinho no RN e a precipitação pluviométrica anual em Areia Branca nos períodos 1938-1948, 1949-1958 e 1959-1968, já que mesmo em anos de tempo meteorológico favorável, os produtores não poderiam comercializar além de sua cota estabelecida pelo governo central. Após o fim das cotas, o quadro muda e no período seguinte 1969-1978 a correlação cresceu de forma considerável $(-0,46)$, passando a ser fraca negativa. Nos períodos seguintes quando o RN passa a dominar quase todo o mercado nacional a correlação passa a ser moderada negativa.

Devido ao seu alto potencial produtivo, primeiramente apenas natural (DINIZ; VASCONCELOS, 2017), e posteriormente também devido à técnica (DINIZ; VASCONCELOS; MARTINS, 2015), durante muitos anos os produtores potiguares mantiveram sua produção de sal marinho em uma quantidade muito aquém de suas possibilidades, por isso a influência da precipitação registrada só é mais marcante em anos de chuvas acima de $50 \%$ da média como as do ano de 1985, quando foram registrados $2.066 \mathrm{~mm} /$ ano de chuvas em Mossoró, $2.194 \mathrm{~mm} / \mathrm{ano}$ em Areia Branca e $1780 \mathrm{~mm} / \mathrm{ano}$ em Macau (SUDENE, 1990). Neste ano a produção foi reduzida para menos da metade do ano anterior no Estado do Rio Grande do Norte, decaindo de 3.126.519 t em 1984 para 1.339.874 t em 1985 (Gráfico 2). O coeficiente de correlação linear de Pearson ( $r$ ) entre pluviometria e produção de sal marinho não é maior no RN porque se, por exemplo, em um ou dois anos ocorre uma grande estiagem no estado mesmo que o terceiro também seja de estiagem, a produção tende a ser baixa, o que se deve aos estoques feitos nos anos anteriores por parte dos empresários. Esse é outro fato que contribui para uma menor correlação entre clima e produção de sal no RN, considerados longos períodos.

A correlação da produção de sal marinho no RN é maior com o crescimento da economia brasileira do que com a precipitação pluviométrica anual, caso sejam considerados períodos maiores.

\section{Correlação entre a economia brasileira e a produção de sal marinho}

Relacionou-se a produção brasileira de sal marinho com o Produto Interno Bruto (PIB) do país, considerando que o sal é um produto indispensável à indústria. Para tal correlação, utilizou-se o mesmo Coeficiente de Correlação Linear de Pearson (r). Os resultados (Quadro 3) mostram 
que existe correlação forte positiva $(r=0,94)$ entre a produção brasileira de sal marinho e o PIB do país entre os anos de 1938 e 2000. A mesma correlação forte positiva $(r=0,95$, Gráfico 3 , Quadro 3) é percebida quando cruzados os dados da produção do RN com o PIB do Brasil. Entretanto, ao correlacionar as mesmas produções de Brasil e RN entre os anos de $2000 \mathrm{e}$ 2013 a correlação encontrada é moderada positiva $(r=0,59$ e $r=0,56$ respectivamente).

Gráfico 3 - Dispersão da produção de sal marinho no Rio Grande do Norte em tone ladas e PIB do Brasil em milhões de reais a preços de 1999 entre 1938 e 2000.

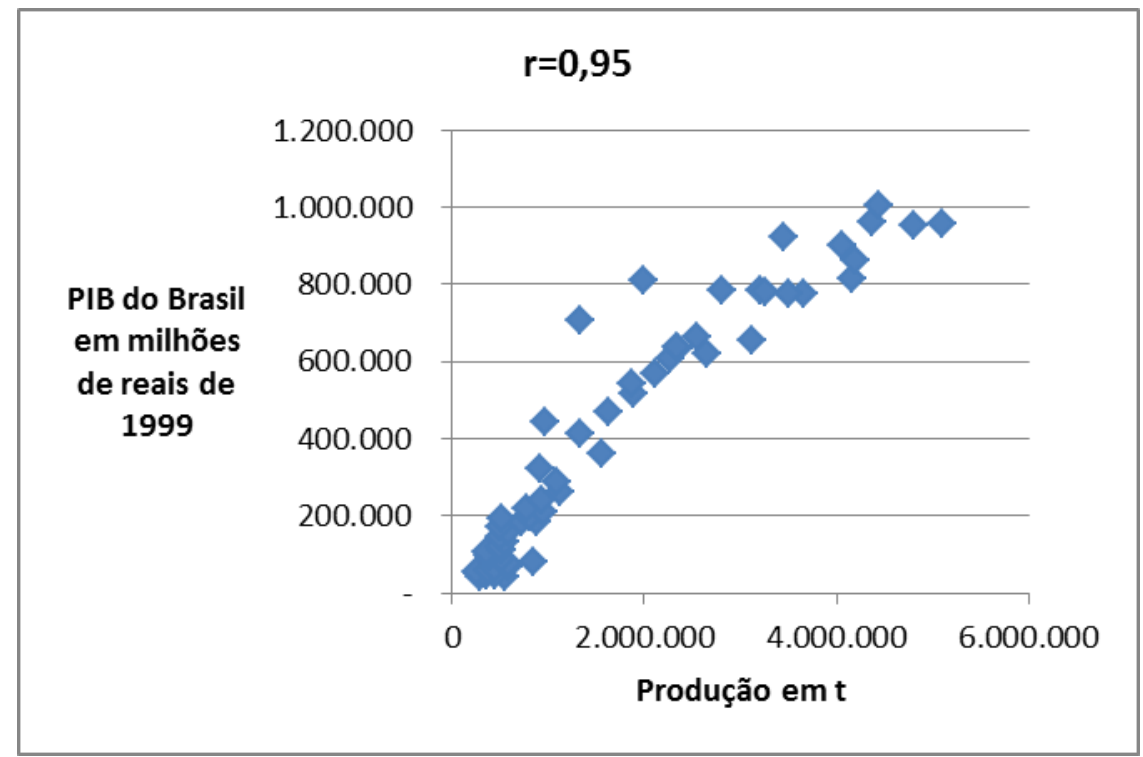

Fonte - Dados da produção de sal - Anuário Estatístico do Brasil - anos 1941/1945 a 1987/1988;e Sumário Mineral Brasileiro anos de 1991 a 2001. Dados do PIB do Brasil em R\$ a preços de 1999 (IBGE, 2006). Não há dados de produção de sal para o ano de 1939.

Quadro 3 - Coeficiente de correlação linear de Pearson (r) entre a produção de sal marinho no Brasil, no RN, no CE e no RJ; e o PIB do Brasil entre os anos de 1938 e 2000 e de 2000 a 2013.

\begin{tabular}{|c|c|c|c|c|}
\hline Períodos & r no Brasil & r no RN & r no CE & r no RJ \\
\hline $\mathbf{1 9 3 8 - 2 0 0 0}$ & 0,94 & 0,95 & 0,09 & 0,40 \\
\hline $\mathbf{2 0 0 0 - 2 0 1 3}$ & 0,59 & 0,56 & 0,80 & 0,24 \\
\hline
\end{tabular}

Fonte - Dados da produção de sal: Anuário Estatístico do Brasil - anos 1941/1945 a 1990; e Sumário Mineral Brasileiro de 1991 a 2009. Dados do PIB do Brasil entre 1938 e 2000 em R\$ a preços de 1999 (IBGE, 2006). Dados do PIB do Brasil entre 2000 e 2011 a preços correntes (IBGE, online). Não há dados de produção de sal para o ano de 1939

Desde o início do século $X X$, a produção brasileira de sal marinho se dedicou principalmente a atender ao mercado nacional, alguns autores comentam ter havido alguns períodos após 1938 em que houve importações (CARVALHO JÚNIOR; FELIPE; ESCÓSSIA, 1982). Entretanto, na maior parte do século $X X$ o Brasil produzia sal suficiente para atender quase $100 \%$ de seu mercado, havendo ainda alguns excedentes para a exportação, por exemplo, em 1994 a balança comercial do produto era francamente favorável ao país, uma vez que foram importadas nesse ano 12.000 toneladas, enquanto que foram exportadas 277.000 toneladas (DNPM, 1997; Gráfico 4). 
Gráfico 4 - Importação e exportação de sal do Brasil entre os anos de 1989 e 2013.

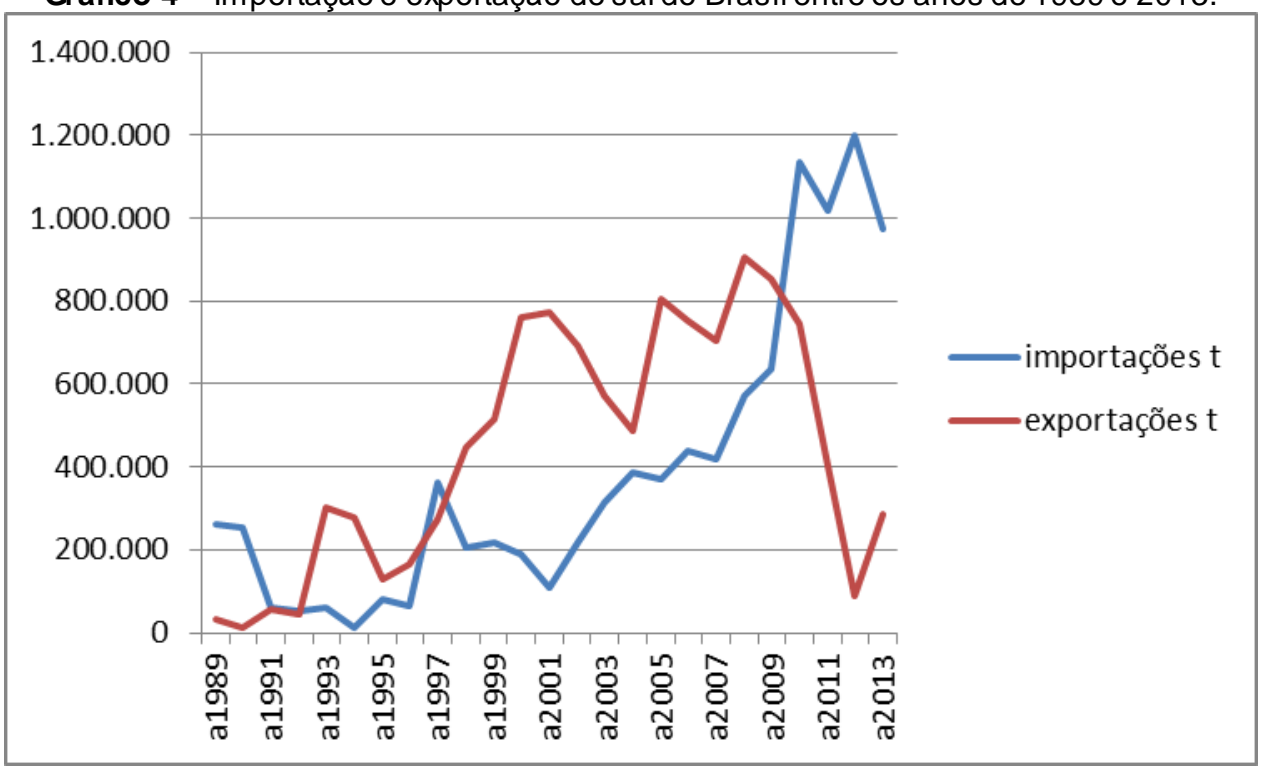

Fonte - Sumário Mineral Brasileiro anos de 1990 a 2014.

O porto de Areia Branca é o canal de exportação que leva o sal das salinas do RN e CE para países como Estados Unidos e Nigéria. Esse porto se dedica, atualmente, exclusivamente à exportação de sal marinho, já que o mercado interno é abastecido utilizando principalmente o transporte rodoviário.

Como é possível visualizar no Gráfico 4, desde o ano de 2001, a importação de sal para o Brasil tem crescido de forma exponencial. Eram importadas 110 mil t em 2001 contra 1.134 mil $\mathrm{t}$ em 2010, um crescimento de mais de dez vezes em dez anos. O sal proveniente principalmente do Chile tem aportado em Santos e nos estados do Sul do país com preços inferiores ao sal produzido no Rio Grande do Norte e Ceará. Os produtores brasileiros reclamam de concorrência desleal, já que os navios que levam minério de ferro do Brasil ao país andino retornam trazendo o sal deste país como lastro nos navios. Esse sal paga tarifa de frete quase irrisória, o que tem aumentado muito a entrada do produto chileno no Brasil.

No ano de 2009 o Brasil já importava 634.783 t de sal, sendo cerca de $98 \%$ de origem chilena (DNPM, 2010). Do ano de 2009 para 2010 houve outro grande crescimento de $79 \%$, sendo importados 1,1 milhão de t. A quantidade importada permaneceu praticamente a mesma em 2011(DNPM, 2012), havendo pequeno decréscimo. Vale lembrar que esse número era apenas 12 mil t em 1994, registrando um crescimento de quase 100 vezes num período de 16 anos, de maneira que em 2010, 99\% do sal importado foi de procedência chilena (DNPM, 2011).

As exportações de sal cresceram bem menos, e se considerarmos apenas os últimos anos, pode-se afirmar que elas vêm diminuindo. Em 2008, foram mais de 900 mil t, passando a ser de pouco mais de 850 mil t em 2009, 754 mil t em 2010, 402 mil t em 2011 e para ínfimos 89.908 t em 2012. (DNPM, 2010, 2011 e 2012) t. A quantidade exportada em 2012 representa um decrescimento de mais de três vezes em relação a 1993 (de 303 mil t para 89 mil t). A crise internacional tem impactado no setor salineiro, já que no início da década são ruins os números apresentados pela indústria de países importadores de sal como os EUA. Se considerarmos o mesmo período (1994-2011) o crescimento das importações foi de mais de 100 vezes. Esses números explicam o motivo pelo qual a atividade salineira não tem crescido nos últimos anos no Brasil. O crescimento da demanda interna tem sido atendido pelo sal chileno.

Ao contrário da produção de sal marinho no Brasil, nos últimos anos, o consumo interno de sal é crescente. De 5,7 milhões de t, em 2009 para 8,5 milhões de t em 2012, e o principal destino é a indústria química que consome $33 \%$ do sal: 
[...] outros setores consumidores de sal foram: consumo humano e animal, agricultura e alimentos, que, por estimativa, responderam com 2,23 milhões de $t(30,1 \%)$; outros setores, como frigoríficos, curtumes, charqueadas, indús trias têxtil e farmacêutica, pros pecção de petróleo e tratamento d'água, responderam com 2,25 milhões de $\mathrm{t}(30,3 \%)$. A indústria em geral e distribuidores responderam pelas $487 \mathrm{mil} \mathrm{t}(6,6 \%)$ restantes (DNPM, 2011, p. 2).

Atendendo às demandas dos produtores brasileiros de sal, o Governo Federal editou medidas antidumping para diminuir a concorrência desleal. $O$ ato se deu através da Resolução no 61 de 08/09/2011 da Câmara de Comércio Exterior - CAMEX Segundo o Ministério da Indústria e Comércio Exterior - MDIC a CAMEX:

[...] aprovou a aplicação de direito antidumping sobre importações de sal grosso do Chile (NCM 2501.00.19) utilizado na fabricação dos seguintes produtos (intermediários ou finais): cloro líquido, ácido clorídrico, hipoclorito de sódio, dicloroetano, soda cáustica, clorato de sódio ou carbonato de sódio. O sal grosso objeto da medida não é aplicado ao consumo humano nem animal. A medida vale por cinco anos e será recolhida sob a forma de alíquota ad valorem de $35,4 \%$.

Também foi homologado compromisso de preços para amparar as importações brasileiras de sal grosso fabricado e exportado pela empresa chilena Sociedad Punta de Lobos S.A. Com a decisão da Camex, o Brasil chega a 81 medidas de defes a comercial em vigor aplicadas (BRASIL, 2011, online).

A ação antidumping parece ter surtido efeito, depois de seguidas altas nas importações desde o ano de 2001. Entre 2010 e 2011 houve tímida queda nas importações de sal por parte do Brasil de 1.134 mil t para 1.118 mil t, em 2012 houve novo aumento para $1.197 \mathrm{t}$, mas em 2013 houve novo decréscimo para 972 mil t (Gráfico 4). Nos próximos anos se terá a exata noção do efeito da medida antidumping, contudo, os dados atuais permitem depreender pequeno decréscimo no crescimento num período de quatro anos. Após a medida antidumping de 2011 a economia salineira do Brasil voltou a crescer nos anos de 2012 e 2013.

Analisando o período 1938-2000, percebe-se que a correlação da produção de sal marinho no segundo e terceiro maiores estados produtores, respectivamente RJ e CE, é fraca positiva em ambos. Analisando outros períodos mais específicos (Quadro 4) é possível perceber que a correlação entre a produção de sal no CE e o PIB do Brasil foi moderada positiva desde 1938 até 1969, mais ou menos quando tem início o declínio da economia salineira cearense, passando a ter correlação ínfima positiva no período 1970-2000. Já em relação ao RJ, a correlação verificada entre sua produção salineira e o PIB brasileiro foi fraca positiva nos períodos 1938-2000 e 2000-2011 (Quadro 2) e moderada positiva nos períodos mais específicos considerados (Quadro 3).

Quadro 4 - Coeficiente de correlação linear de Pearson ( $r$ entre a produção de sal marinho no Brasil, no RN, no CE e no RJ; e o PIB do Brasil entre os anos de 1938 a 1969 e de 1970 a 2000.

\begin{tabular}{|c|c|c|c|c|}
\hline Períodos & r no Brasil & r no RN & r no CE & r no RJ \\
\hline $\mathbf{1 9 3 8 - 1 9 6 9}$ & 0,84 & 0,78 & 0,75 & 0,76 \\
\hline $\mathbf{1 9 7 0 - 2 0 0 0}$ & 0,80 & 0,90 & 0,02 & 0,74 \\
\hline
\end{tabular}

Fonte - Dados da produção de sal - Anuário Estatístico do Brasil -anos 1941/1945 a 1990; e Sumário Mineral Brasileiro de 1991 a 2001. Dados do PIB do Brasil entre 1938 e 2000 em R\$ a preços de 1999 (IBGE, 2006). Não há dados de produção de sal para o ano de 1939.

No Gráfico 5 é possível perceber que a produção de sal marinho no Rio Grande do Norte e no Brasil cresceu em um ritmo até maior que o da economia brasileira entre os anos de $1938 \mathrm{e}$ 2000, o que pode ser verificado especialmente entre as décadas de 1970 e 1990. 
Gráfico 5 - Produção de sal marinho no Rio Grande do Norte (RN) e no Brasil (BRA) em tone ladas e PIB do Brasil em milhões de reais de 1999 entre 1938 e 2000.

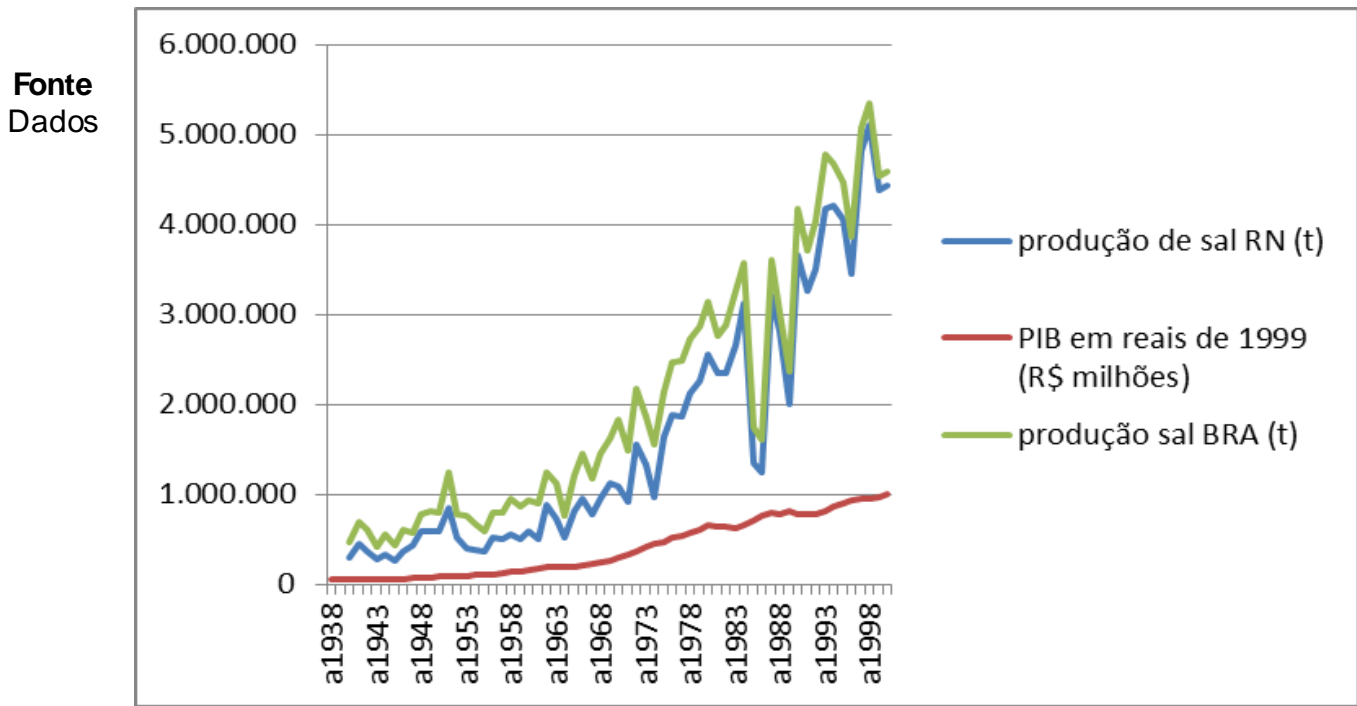

produção de sal: Anuário Estatístico do Brasil - anos 1941/1945 a 1987/1988; e Sumário Mineral Brasileiro anos de 1991 a 2001. Dados do PIB do Brasil: IBGE. Não há dados de produção de sal para o ano de 1939.

No Gráfico 6, é nítido perceber que do ano 2000 ao ano de 2013 a economia brasileira vem crescendo em um ritmo muito maior que o da produção de sal marinho do RN e do Brasil. Essa produção esteve praticamente estagnada no período, com pequena recuperação que parece ter se iniciado em 2012. Se tomarmos, por exemplo, apenas o maior produtor de sal marinho do Brasil, no RN eram produzidas 4.436 mil t em 2000 e 4.500 mil t de sal marinho em 2011, crescimento quase nulo no período. Nesse mesmo espaço de tempo, o PIB do Brasil cresceu mais de três vezes e meia passando de 1.199 .092 milhões de $R \$$ para 4.373 .658 milhões de $\mathrm{R} \$$ em moeda corrente (a preços de 2016). O forte crescimento das importações (Gráfico 6) foi responsável por atender à crescente demanda por sal marinho da economia brasileira, entre 2001 e 2011, a partir de 2012 diminuíram as importações e voltou a crescer a produção nacional.

Gráfico 6 - Produção de sal marinho no Rio Grande do Norte (RN) e no Brasil (BRA) em toneladas e PIB do Brasil a preços correntes em milhões de reais entre 2000 e 2013.

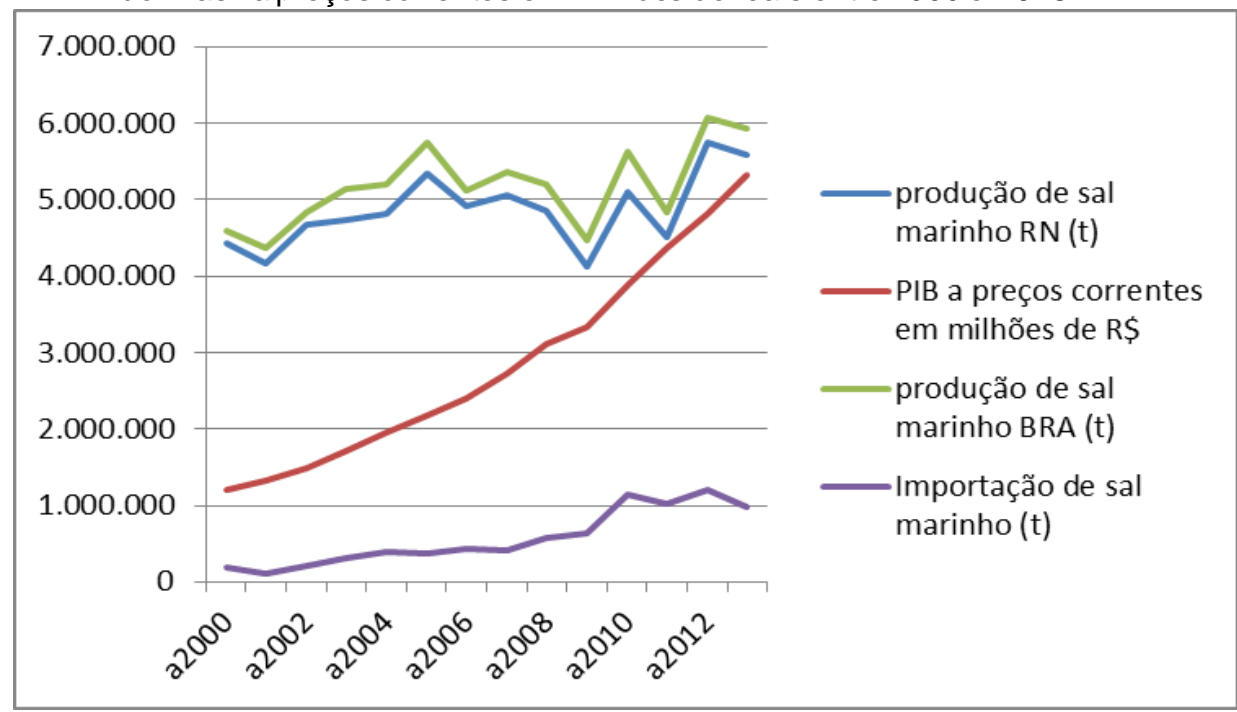

Fonte - Dados da produção de sal: Sumário Mineral Brasileiro anos de 2001 a 2014. Dados do PIB do Brasil:IBGE. 


\section{CONSIDERAÇÕES FINAIS}

A década de 1970 trouxe grandes mudanças para a produção de sal no Nordeste, notadamente, no Estado do Rio Grande do Norte. Em 1974, foi construído e inaugurado o Porto-llha, um terminal portuário salineiro em Areia Branca, que viria a mudar radicalmente a relação custo de produção versus frete do sal, favorecendo a produção e baixando o frete, consideravelmente. Outros fatores positivos foram a melhoria no sistema rodoviário brasileiro e a mecanização da indústria salineira potiguar. Os produtores norte-rio-grandenses iniciaram um processo de crescimento econômico que culminou com a criação de um oligopólio que passou a dominar mais de $90 \%$ da produção de sal marinho no Brasil.

A ascensão da economia salineira potiguar causou forte impacto nas salinas de outros estados brasileiros que viram suas produções locais desmanteladas pela entrada do sal potiguar em todas as unidades da federação. Hoje, o sal potiguar é mais barato e de melhor qualidade que o restante do sal produzido no Brasil. Atualmente, além do Rio Grande do Norte, apenas Ceará, Piauí e Rio de Janeiro persistem produzindo sal marinho, sendo que nos dois primeiros estados, as empresas produtoras pertencem a grupos empresariais com sede no Rio Grande do Norte.

Desde os anos 2000 o oligopólio dos produtores potiguares se encontra ameaçado pela concorrência do sal chileno, a concorrência predatória do produto daquele país foi responsável pela estagnação da economia salineira brasileira e potiguar até o ano de 2011, tendo sido retomado o crescimento da produção nacional a partir de 2012.

Constata-se que existe forte influencia da precipitação pluviométrica anual com 0 desenvolvimento da atividade salineira, que é concentrada, principalmente, em determinada porção do Nordeste Setentrional, nessa região a atividade é influenciada pela variação interanual do regime de chuvas.

\section{REFERÊNCIAS}

ANDRADE, M. C. de. O território do sal. Mossoró: Coleção Mossoroense, 1995.

BRASIL. Ministério da Indústria e Comércio Exterior - MDIC. Camex aprova antidumping para tubos de aço carbono da China e sal grosso do Chile. Assessoria de comunicação social do MDIC, Brasília, nov. 2011. Disponível em:

$<$ http://www.desenvolvimento.gov.br/sitio/interna/noticia. $p h p$ ?area=1\&noticia=10972>. Acesso em: 10 abr. 2013.

CARVALHO JÚNIOR, J. V. de; FELIPE, J. L. A.; ESCÓSSIA, C. A. da. Introdução à história do sal. Terra e Sal, Mossoró, ano 1, n.2, p. 6-26, dez. 1982.

DNPM. Departamento Nacional de Produção Mineral. Sumário Mineral. Brasília: DNPM/DIPLAM, 1997.

Anuário Mineral Brasileiro. Brasília: DNPM, 1995.

Sumário Mineral. Brasília: DNPM/DIPLAM, 1998.

Sumário Mineral. Brasília: DNPM/DIPLAM, 1999.

Sumário Mineral. Brasília: DNPM/DIPLAM, 2000.

Sumário Mineral. Brasília: DNPM/DIPLAM, 2001.

$\begin{array}{llllll}\text { Caminhos de Geografia } & \text { Uberlândia-MG } & \text { v. 20,n.69 } & \text { Março/2019 } & \text { p. 20-35 } & \text { Página } 31\end{array}$


Sumário Mineral. Brasília: DNPM/DIPLAM, 2002.

Sumário Mineral. Brasília: DNPM/DIPLAM, 2003.

Sumário Mineral. Brasília: DNPM/DIPLAM, 2004a.

Anuário Mineral Brasileiro. Brasília: DNPM, 2004b.

Sumário Mineral. Brasília: DNPM/DIPLAM, 2005.

Sumário Mineral. Brasília: DNPM/DIPLAM, 2006.

Sumário Mineral. Brasília: DNPM/DIPLAM, 2007.

Sumário Mineral. Brasília: DNPM/DIPLAM, 2008.

Sumário Mineral. Brasília: DNPM/DIPLAM, 2009.

Sumário Mineral. Brasília: DNPM/DIPLAM, 2010.

Sumário Mineral. Brasília: DNPM/DIPLAM, 2011.

Sumário Mineral. Brasília: DNPM/DIPLAM, 2012.

Sumário Mineral. Brasília: DNPM/DIPLAM, 2013.

https://doi.org/10.20336/rbs. 185

Sumário Mineral. Brasília: DNPM/DIPLAM, 2014.

https://doi.org/10.20336/rbs. 186

DINIZ, M. T. M.; VASCONCELOS, F. P.; MARTINS, M. B. Inovação tecnológica na produção brasileira de sal marinho e as alterações sócioterritoriais dela decorrentes: uma análise sob a ótica da teoria do empreendedorismo de schumpeter. Sociedade e Natureza, Uberlândia, v. 27, n. 3, p. 421-438, set.-dez. 2015. https://doi.org/10.1590/1982-451320150305

DINIZ, M. T. M.; VASCONCELOS, F. P. Aspectos históricos e dinâmica locacional da economia salineira brasileira. Boletim Gaúcho de Geografia., v.43, p.1 - 26, 2016.

CONDICIONANTES NATURAIS À PRODUÇÃO DE SAL MARINHO NO BRASIL. MERCATOR (FORTALEZA. ONLINE). , v.16, p.1 - 19, 2017.

https://doi.org/10.4215/rm2017.e16013

EMPARN. Empresa de Pesquisa Agropecuário do Rio Grande do Norte. Meteorologia: Monitoramento pluviométrico. Disponível em:

<http://189.124.135.176/monitoramento/monitoramento.php>. Acesso em: 19 abr. 2013.

FUNCEME. Fundação Cearense de Meteorologia e Recursos Hídricos. Download de séries históricas. Disponível em: <http://www.funceme.br/index.php/areas/tempo/download-de-serieshistoricas >. Acesso em: 19 abr. 2013. 
IBGE. Instituto Brasileiro de Geografia e Estatística. Anuário Estatístico do Brasil 1941/1945. Rio de Janeiro: IBGE, 1945.

Anuário Estatístico do Brasil 1941/1945. Rio de Janeiro: IBGE, V.6, 1946.

Anuário Estatístico do Brasil 1946. Rio de Janeiro: IBGE, V.7, 1947.

Anuário Estatístico do Brasil 1947. Rio de Janeiro: IBGE, V.8, 1948.

Anuário Estatístico do Brasil 1948. Rio de Janeiro: IBGE, V.9, 1949.

Anuário Estatístico do Brasil 1949. Rio de Janeiro: IBGE, V.10, 1950.

Anuário Estatístico do Brasil 1950. Rio de Janeiro: IBGE, V.11, 1951.

Anuário Estatístico do Brasil 1951. Rio de Janeiro: IBGE, V.12, 1952.

Anuário Estatístico do Brasil 1952. Rio de Janeiro: IBGE, V.13, 1953.

Anuário Estatístico do Brasil 1953. Rio de Janeiro: IBGE, V.14, 1953.

Anuário Estatístico do Brasil 1954. Rio de Janeiro: IBGE, V.15, 1954.

Anuário Estatístico do Brasil 1955. Rio de Janeiro: IBGE, V.16, 1955.

Anuário Estatístico do Brasil 1956. Rio de Janeiro: IBGE, V.17, 1956.

Anuário Estatístico do Brasil 1957. Rio de Janeiro: IBGE, V.18, 1957.

Anuário Estatístico do Brasil 1958. Rio de Janeiro: IBGE, V.19, 1958.

Anuário Estatístico do Brasil 1959. Rio de Janeiro: IBGE, V.20, 1959.

Anuário Estatístico do Brasil 1960. Rio de Janeiro: IBGE, V.21, 1960.

Anuário Estatístico do Brasil 1961. Rio de Janeiro: IBGE, V.22, 1961.

Anuário Estatístico do Brasil 1962. Rio de Janeiro: IBGE, V.23, 1962.

Anuário Estatístico do Brasil 1963. Rio de Janeiro: IBGE, V.24, 1963.

Anuário Estatístico do Brasil 1964. Rio de Janeiro: IBGE, V.25, 1964.

Anuário Estatístico do Brasil 1965. Rio de Janeiro: IBGE, V.26, 1965.

Anuário Estatístico do Brasil 1966. Rio de Janeiro: IBGE, V.27, 1966.

Anuário Estatístico do Brasil 1967. Rio de Janeiro: IBGE, V.28, 1967. 
Anuário Estatístico do Brasil 1968. Rio de Janeiro: IBGE, V.29, 1968.

Anuário Estatístico do Brasil 1969. Rio de Janeiro: IBGE, V.30, 1969.

Anuário Estatístico do Brasil 1970. Rio de Janeiro: IBGE, V.31, 1970.

Anuário Estatístico do Brasil 1971. Rio de Janeiro: IBGE, V.32, 1971.

Anuário Estatístico do Brasil 1972. Rio de Janeiro: IBGE, V.33, 1972.

Anuário Estatístico do Brasil 1973. Rio de Janeiro: IBGE, V.34, 1973.

Anuário Estatístico do Brasil 1974. Rio de Janeiro: IBGE, V.35, 1974.

Anuário Estatístico do Brasil 1975. Rio de Janeiro: IBGE, V.36, 1975.

Anuário Estatístico do Brasil 1976. Rio de Janeiro: IBGE, V.37, 1976.

Anuário Estatístico do Brasil 1977. Rio de Janeiro: IBGE, V.38, 1977.

Anuário Estatístico do Brasil 1978. Rio de Janeiro: IBGE, V.39, 1978.

Anuário Estatístico do Brasil 1979. Rio de Janeiro: IBGE, V.40, 1979.

Anuário Estatístico do Brasil 1980. Rio de Janeiro: IBGE, V.41, 1980.

Anuário Estatístico do Brasil 1981. Rio de Janeiro: IBGE, V.42, 1981.

Anuário Estatístico do Brasil 1982. Rio de Janeiro: IBGE, V.43, 1982.

Anuário Estatístico do Brasil 1983. Rio de Janeiro: IBGE, V.44, 1983.

Anuário Estatístico do Brasil 1984. Rio de Janeiro: IBGE, V.45, 1984.

Anuário Estatístico do Brasil 1985. Rio de Janeiro: IBGE, V.46, 1985.

Anuário Estatístico do Brasil 1986. Rio de Janeiro: IBGE, V.47, 1986.

Anuário Estatístico do Brasil 1987/1988. Rio de Janeiro: IBGE, V.48, 1988.

Anuário Estatístico do Brasil 1989. Rio de Janeiro: IBGE, V.49, 1989.

Anuário Estatístico do Brasil 1990. Rio de Janeiro: IBGE, V.50, 1990.

Anuário Estatístico do Brasil 1991. Rio de Janeiro: IBGE, V.51, 1991.

Anuário Estatístico do Brasil 1992. Rio de Janeiro: IBGE, V.52, 1992.

Anuário Estatístico do Brasil 1993. Rio de Janeiro: IBGE, V.53, 1993.

\begin{tabular}{llllll}
\hline Caminhos de Geografia & Uberlândia -MG & v. 20, n.69 & Março/2019 & p. 20-35 & Página 34
\end{tabular}


Anuário Estatístico do Brasil 1994. Rio de Janeiro: IBGE, V.54, 1994.

. Anuário Estatístico do Brasil 1995. Rio de Janeiro: IBGE, V.55, 1995.

Anuário Estatístico do Brasil 1996. Rio de Janeiro: IBGE, V.56, 1996.

Anuário Estatístico do Brasil 1997. Rio de Janeiro: IBGE, V.57, 1997.

Estatísticas do Século XX Rio de Janeiro: IBGE, 2006.

Downloads: Estatísticas. Disponível em:

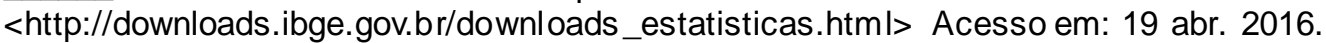

NAGHETTINI, M.; PINTO, E. J. de A. Hidrologia estatística. Belo Horizonte: CPRM, 2007.

SOUZA, A. Coeficiente de correlação linear de Pearson. Universidades dos Açores.

Departamento de Matemática. Ilha de São Miguel. Disponível em:

<http://www.aurea.uac.pt/pdf_MBA/coef_correl_Pearson.pdfs. Acesso em: 10 out. 2012.

SUDENE. Superintendência do Desenvolvimento do Nordeste. Dados pluviométricos mensais do Nordeste. Superintendência do Desenvolvimento do Nordeste, Recife, 1990 (Série Pluviometria 1 a 10).

Recebido em: 16/11/2017

Aceito para publicação em: 21/03/2019 